\title{
POPULATION GENETICS OF PARTIAL MALE-STERILITY AND THE EVOLUTION OF MONOECY AND DIOECY
}

\author{
D. CHARLESWORTH and B. CHARLESWORTH \\ School of Biological Sciences, University of Sussex, Falmer, Sussex BN1 $9 Q G$
}

Received 13.xii.77

\begin{abstract}
SUMMARY
Selection on reproductive phenotype in plants is studied, using a theoretical model. Fitnesses of mutant phenotypes with altered male and female fertility are derived, assuming an initially hermaphrodite or monoecious population with no self-incompatibility mechanism, with partial self-fertilisation, and with some inbreeding depression. These fitness expressions are used to derive conditions for the spread of such mutations, in terms of the minimum increase in opposite-sex fertility that will give a phenotype with reduced male or female fertility a selective advantage over the original type, taking into account the effect on the selfing rate of altering the sex phenotype. Conditions for polymorphism are also obtained. The conditions are used to study the evolution of dioecy from monoecy, and the evolution of gynomonoecy and monoecy from the hermaphrodite state.
\end{abstract}

\section{INTRODUCTION}

IN an earlier paper, we have examined the possibility of the evolution of dioecy from the hermaphrodite state via gynodioecy (Charlesworth and Gharlesworth, 1978). It seems likely that dioecy has also evolved from monoecy. Lewis (1942) showed that dioecy " is more frequently associated with monoecy than with hermaphroditism" in the British flora. Sporadic cases of dioecy in predominantly monoecious families are not common, however. In the British flora, there are Mercurialis annua and perennis, and Urtica dioica. In families with hermaphrodite, monoecious and dioecious species, only Carex dioica belongs to a wholly monoecious genus, and Antennaria dioica is closely related to a monoecious species. The situation seems to be similar in other floras. Baker (1959) states that, although monoecy and dioecy are often found in the same families, there is very little tendency for them to occur in the same genus. Some of the associations that occur may therefore be due to a common potentiality for the formation of unisexual flowers, or to evolution of monoecy from dioecy (Baker, 1959).

The best data on this subject were obtained by Lloyd $(1972,1975 a, b)$ in Cotula. There is evidence for the gradual evolution of dioecy from monoecy (and for evolution of monoecy from dioecy). For example, in seven dioecious species in section Elongata, there is a low frequency of plants with bisexual heads. Lloyd (1975a) points out that " the virtual absence of sterile neuter florets and the presence of functional minority-sex florets in both sexes confirm that sterility genes have not participated in the evolution of dioecy in Cotula". It therefore seems that dioecy can evolve from monoecy by a gradual process of alteration of the relative proportions of male and female flowers, rather than via gynodioecy with essentially 
complete male-sterility of the females. When dioecy has evolved from monoecy, we would expect both sexes to show intermediate-sex forms, as in Cotula and Mercurialis annua, whereas when gynodioecy is an intermediate stage in the evolution of dioecy the females should be completely, or very nearly, male sterile, while the " males" will often be much more variable (Charlesworth and Charlesworth, 1978). This is the case in many species, for example Cirsium arvense (Lloyd and Myall, 1976, see also Westergaard, 1958).

There are two questions which should be studied in view of these data. First, how can dioecy be established in a monoecious species, and what is the role of avoidance of selfing in the process? Second, what special features would we expect in dioecy that has evolved from monoecy, compared with that derived from a hermaphrodite species? To try to answer these questions, we examine the conditions for the establishment of phenotypes with partial alterations in male and/or female fertility, using the method of Charlesworth and Charlesworth (1978) for calculating fitnesses in a partially selfing population.

In a species with no self-incompatibility system, changes in the selfing rate could be produced by any of the following mechanisms: changes in the relative times of maturity of the male and female reproductive organs, changes in the morphological arrangement of the flower so that selfpollination is promoted or avoided, or changes in the amount or quality of the pollen. All these changes are likely to affect outcrossing ability, as well as the rate of selfing. For instance, a change in the timing of pollen maturity could reduce the chance of selfing, but would be very likely to reduce the ability to pollinate other individuals. Changes in pollen quality or quantity will of course have similar effects, and so may changes in the anther or stigma positions (Charlesworth and Charlesworth, 1978). Formally, all these possibilities can be treated as changes in male or female fertility, but complete male or female sterility is unlikely to occur, except in the case of changes in the amount or quality of the pollen. Male-fertility changes are considered first, in what follows; we then examine how selection on mutants with reduced female fertility would be affected by the presence of a femalelike form (with reduced male fertility) in the population. The conclusions that can be drawn from these theoretical results can also be used to examine the conditions under which monoecy can evolve from hermaphroditism; this topic is discussed in Section 5.

\section{Model AND Assumptions}

The model studied is an extension of that considered previously (Charlesworth and Charlesworth, 1978); the definition of fitness that we shall employ is explained in detail in that paper. The general formula for the fitness of the $i$ th form, with male fertility $b_{i}$, female fertility $e_{i}$, and selfing rate $s_{i}$, is given by:

$$
w_{i}=\frac{e_{i}\left[1+s_{i}(1-2 \delta)\right]+U b_{i} / V}{2 W}
$$

where $U, V$ and $W$ depend on the frequencies $\left(z_{i}\right)$ and phenotypes of the members of the population, as follows: 


$$
\begin{aligned}
U & =\Sigma\left(1-s_{i}\right) e_{i} z_{i} \\
W & =U+\Sigma s_{i}(1-\delta) e_{i} z_{i} \\
V & =\Sigma b_{i} z_{i}
\end{aligned}
$$

and $\delta$ is the inbreeding depression (equivalently, $1-\delta$ is the viability of a product of selfing, relative to that of an outcrossed progeny). This general formula for fitness will be used in what follows, substituting values for the $e_{i}, b_{i}$ and $s_{i}$, according to the model being considered.

A number of assumptions have been made which are features of all the models to be studied below. First, the ovules of all plants with female reproductive potential are assumed to be fertilised to the same extent. For example, when considering the spread of a form with reduced pollen production in an initially monoecious population, we assume that the chance of fertilisation of the ovules of the new form is unchanged, and also that there is sufficient excess pollen that fertilisation is not affected as such a form spreads in the population. Lloyd $(1974,1975 a, b, c)$ gives a treatment of models of male-sterility mutations without this assumption, but allowing fertilisation rates to depend on the frequencies of male and female flowers in the population. A second assumption, which we have discussed previously (Charlesworth and Charlesworth, 1978), is that the production of flowers consumes resources, so that for example a reduction in pollen production could release energy or material for increased production of ovules. This is envisaged as a purely physiological effect, and was first proposed, and termed "compensation", by Darwin (1877).

\section{Selegtion for Reduced male Fertility}

Consider a population initially consisting of plants with both male and female reproductive potential, i.e. either hermaphrodite or monoecious. We will first investigate the conditions for the spread of a new form with lowered male fertility, due either to decreased pollen output, or (in a monoecious species) to a change in the frequency of male flowers. Let the pollen output of the new form be $(1-K) b$, where $b$ is the pollen output of the original form. Let the selfing rate of the original form be $s$, and let its female fertility be arbitrarily given the value 1 . If the new form has selfing rate $s$, and female fertility $1+k$, its fitness (by substituting into Eq. (1)) is:

$$
w_{f}=\frac{(1+k)\left[1+s_{f}(1-2 \delta)\right]+U b(1-K) / V}{2 W} .
$$

If the population consists overwhelmingly of the fully male-fertile form, we can use the values of $U, V$ and $W$ calculated for a population of this form alone. We then have:

$$
\begin{aligned}
& U=1-s \\
& W=1-s \delta \\
& V=b
\end{aligned}
$$


giving :

$$
w_{f}=\frac{(1+k)\left[1+s_{f}(1-2 \delta)\right]+(1-K)(1-s)}{2(1-s \delta)} .
$$

This generalises the result of Charlesworth and Charlesworth (1978) to the case of a mutation causing partial reduction in male fertility. When $K$ is put equal to 1 (i.e. complete male sterility, so that $s_{f}$ must be set equal to zero as well) the result is identical to that obtained previously for a malesterile mutant.

It seems likely that a phenotype with reduced male fertility would have a reduced rate of selfing, but the two effects need not necessarily be the same size. Eq. (3) enables one to find out how great an effect on selfing will give an advantage to a form with reduced male fertility, given a particular effect on female fertility. The denominator in Eq. (3) is positive. The condition for $w_{f}$ to exceed 1 is therefore equivalent to:

$$
(1+k)\left[1+s_{f}(1-2 \delta)\right]+(1-K)(1-s)-2(1-s \delta)>0
$$

The female fertility of the new form, $(1+k)$, must therefore exceed:

$$
\frac{2(1-s \delta)-(1-K)(1-s)}{1+s_{f}(1-2 \delta)}
$$

if it is to be selected for. Alternatively, the condition for spread can be given in terms of $s_{y}$. The expression is complicated if $k$ is not zero, but it is easy to show for the case when the new and the old forms have the same ovule output (i.e. $k=0$ ) that the condition is:

$$
s_{f}<\frac{s(2 \delta-1)+K(1-s)}{2 \delta-1}
$$

if $\delta>\frac{1}{2}$. The right-hand side of this inequality is less than $s$, but greater than $(1-K) s$, proving that some reduction in the selfing rate is necessary if inbreeding depression is greater than $\frac{1}{2}$ (if $\delta<\frac{1}{2}$, the selfing rate must be increased, which is not likely for a mutant that decreases male fertility); the reduction in selfing rate need not, however, be of the same magnitude as the reduction in male fertility. Some numerical examples are shown in table 1 .

\section{TABLE 1}

Minimum fraction by which the selfing rate must be reduced if a female-like mutant with male fertility $(1-\mathrm{K}) \mathrm{b}$ is to have a selective advantage over a bixsexual form with the same female fertility, selfing rate $\mathrm{s}$, and male fertility $\mathrm{b}$

\begin{tabular}{ccccc}
$\begin{array}{c}\text { Inbreeding } \\
\text { depression } \\
\delta\end{array}$ & $s$ & \multicolumn{3}{c}{ Reduction in male fertility, $K$} \\
\cline { 2 - 4 } 0.55 & 0.4 & $>1$ & 0.25 & 0.75 \\
& 0.8 & 0.250 & 0.625 & $>1$ \\
0.6 & 0.4 & 0.750 & $>1$ & $>1$ \\
& 0.8 & 0.125 & 0.313 & 0.938 \\
0.7 & 0.4 & 0.375 & 0.938 & $>1$ \\
& 0.8 & 0.063 & 0.156 & 0.469 \\
0.8 & 0.4 & 0.250 & 0.625 & $>1$ \\
& 0.8 & 0.041 & 0.104 & 0.313 \\
0.9 & 0.4 & 0.188 & 0.468 & $>1$ \\
& 0.8 & 0.031 & 0.078 & 0.234
\end{tabular}


We will next study the conditions for the maintenance of a polymorphism for a male fertility gene, assuming that there are just two phenotypes (i.e. completely dominant or recessive genes). It is obvious that a male-sterility mutation cannot increase to fixation; the conditions for the spread of such a factor, if fully dominant or recessive, are therefore also the conditions for polymorphism. For male-fertility factors of lesser effect, we have studied the conditions for each form to spread when introduced into a population of the other form. The conditions for the spread of the male-infertile form have been.given above. For the original form to increase in frequency in a population of the male-infertile form, Eq. (1) shows that (with the same parameter values as above) we have

$$
\begin{aligned}
U & =\left(1-s_{f}\right)(1+k) \\
W & =\left(1-s_{f} \delta\right)(1+k) \\
V & =(1-K) b
\end{aligned}
$$

so that the fitness expression

must exceed 1.

$$
w_{0}=\frac{1+s(1-2 \delta)+\left(1-s_{f}\right)(1+k) /(1-K)}{2(1+k)\left(1-s_{f} \delta\right)}
$$

It is difficult to deal with the conditions for polymorphism in the general case given above (Eqs. (3) and (5)). We will assume for purposes of illustration that the effects of the mutation on selfing is the same as its effect on male fertility, i.e. $\mathrm{s}_{f}=(1-K) s$; this simplifies matters considerably. The results derived above show that if the reduction in selfing rate is less than this, the new form has a smaller advantage over the original form (provided that $\delta>\frac{1}{2}$ ). Using this principle, one can at least qualitatively extend the results derived below to cases where $s_{f} \neq(1-K) s$. We then find that a sufficient condition for $w$, to exceed 1 is that the product $s \delta$ exceeds $\frac{1}{2}$. When the male-infertile form has the same female fertility as the original form $(k=0)$, this is also a necessary condition. If $s \delta<\frac{1}{2}$, $w_{f}$ is greater than 1 only if

$$
k>\frac{K(1-2 s \delta)}{1+s(1-K)(1-2 \delta)} .
$$

With $k=0, \mathrm{Eq}$. (5) leads to the condition that for $w_{0}$ to exceed 1 we must have:

$$
1-K<\frac{1}{2 s \delta}
$$

This, together with the condition $s \delta>\frac{1}{2}$, defines the conditions for polymorphism for mutations that do not increase female fertility. For example, with $s=\delta=0 \cdot 8$, there will be a polymorphism if male fertility and the selfing rate are reduced by at least $0 \cdot 2188$. Smaller reductions would spread to fixation. With increased female fertility in the new form $(k>0)$, the conditions for polymorphism depend on the values of $k$ as well as $K$. Table 2 shows the results of calculations of the range of values of $k$ that will give polymorphism, with a variety of values of $s, \delta$ and $K$. If $k$ is too low, the female-like form will be eliminated; if too high, it will go to fixation. Table 2 shows that the range of values of $k$ permitting polymorphism is 
TABLE 2

Range of values of $\mathrm{k}$, the increase in female fertility, that give a selective advantage to a phenotype with selfing rate $(1-\mathrm{K}) \mathrm{s}$ and male fertility $(1-\mathrm{K}) \mathrm{b}$. The original form has selfing rate $\mathrm{s}$ and male fertility $\mathrm{b}$

\begin{tabular}{|c|c|c|c|c|}
\hline \multirow{2}{*}{$\begin{array}{c}\text { Inbreeding } \\
\text { depression } \\
\delta\end{array}$} & \multirow{2}{*}{$\begin{array}{c}\text { Selfing rate } \\
\text { of bisexual form } \\
s\end{array}$} & \multicolumn{3}{|c|}{ Reduction in male fertility and in selfing rate $(K)$} \\
\hline & & $0 \cdot 1$ & $0 \cdot 25$ & 0.5 \\
\hline \multirow[t]{3}{*}{0} & 0.9 & $0 \cdot 055-0.062$ & $0 \cdot 149-0.213$ & $0.345-1 \cdot 111$ \\
\hline & $0 \cdot 6$ & $0.065-0.075$ & $0 \cdot 179-0.278$ & $0.385-1.667$ \\
\hline & $0 \cdot 1$ & $0 \cdot 092-0 \cdot 112$ & $0.233-0.435$ & $0 \cdot 476-10 \cdot 0$ \\
\hline \multirow[t]{3}{*}{$0 \cdot 4$} & $0 \cdot 9$ & $0.024-0.034$ & $0 \cdot 062-0 \cdot 149$ & $0 \cdot 128-1 \cdot 185$ \\
\hline & $0 \cdot 6$ & $0.047-0.060$ & $0 \cdot 119-0 \cdot 235$ & $0 \cdot 245-2 \cdot 111$ \\
\hline & $0 \cdot 1$ & $0 \cdot 090-0 \cdot 112$ & $0.227-0.443$ & $0 \cdot 455-16 \cdot 0$ \\
\hline \multirow[t]{4}{*}{$0 \cdot 6$} & $0 \cdot 8$ & $0.005-0.018$ & $0.011-0.125$ & $0.022-1.625$ \\
\hline & $0 \cdot 6$ & $0.031-0.047$ & $0.077-0.211$ & $0 \cdot 149-2 \cdot 667$ \\
\hline & 0.4 & $0.056-0.074$ & $0.138-0.302$ & $0 \cdot 271-4 \cdot 75$ \\
\hline & $0 \cdot 1$ & $0 \cdot 090-0 \cdot 113$ & $0.223-0.448$ & $0.444-23.5$ \\
\hline \multirow[t]{4}{*}{$0 \cdot 8$} & $0 \cdot 8$ & $0 \quad-*$ & $0 \quad-0.026$ & $0-2 \cdot 25$ \\
\hline & $0 \cdot 6$ & $0.006-0.024$ & $0.014-0 \cdot 171$ & $0 \cdot 024-4.333$ \\
\hline & $0 \cdot 4$ & $0.046-0.066$ & $0 \cdot 096-0 \cdot 296$ & $0.205-8.5$ \\
\hline & $0 \cdot 1$ & $0.089-0.113$ & $0.220-0.454$ & $0.433-46 \cdot 0$ \\
\hline
\end{tabular}

* Indicates that the condition for polymorphism cannot be satisfied in this case.

widest when the selfing rate is low, so that low $s$ makes polymorphism likely, compared with higher values of $s$. Similarly, as might be expected, polymorphism is more likely, the greater the effect on male fertility. Polymorphism is possible when there is no inbreeding depression. Eqs. (3) and (5) yield the following range of $k$ values consistent with polymorphism in this case:

$$
\frac{K}{(1-K) s+1}<k<\frac{K}{(1-K)(2+s)-1} .
$$

Some values are shown in table 2 .

The predictions described above for the spread and polymorphism of male-fertility mutations have been checked by computer runs with phenotypes with differing male and female fertilities under various conditions with respect to $s$ and inbreeding depression. It is not possible to write down a simple explicit expression for the frequency of the female-like form at

TABLE 3

Minimum increases in male or female fertility that must accompany a halving of opposite-sex fertility, in order to give the new form a selective advantage over the unmodified form, in a population segregating for a "female " form with the parameter values given in the body of the table

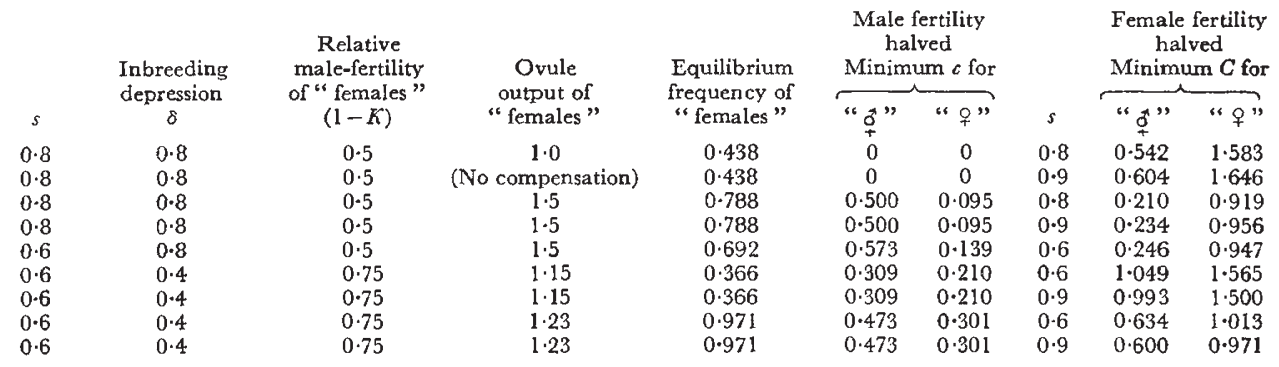


equilibrium, but computer runs show that females can reach any frequency and, as mentioned above, fixation can readily occur. This contrasts with the case of complete male-sterility genes, which it is easy to show cannot rise above a frequency of $\frac{1}{2}$ with nuclear inheritance no matter how great their effect on female fertility (Lloyd, 1974; Charlesworth and Charlesworth, 1978). Some examples of equilibria reached by recessive mutations reducing male fertility are shown in table 3.

\section{INTRODUCTION OF FURTHER GHANGES INTO POPULATIONS CONTAINING "FEMALE" FORMS}

Although we do not know the equilibrium frequency of the female-like form without doing computer calculations, it is nevertheless possible to investigate the fate of further mutations introduced into an equilibrium population containing some frequency of "females ", by using the property that at equilibrium the fitness of the "females" is equal to that of the original form, and also equal to 1 . We can then ask whether a new phenotype, for example a second type with reduced male fertility, will have a fitness higher than that of either of the two forms present in the equilibrium population. We expect that the fitness of such a form would decrease, the higher the frequency of the female-like form already present, and that conversely the fitness of a form with decreased female fertility (and increased male fertility) would be an increasing function of the frequency of females, other things being equal. It has been possible to prove these points for certain particular cases.

\section{(i) Further reductions in male fertility}

Let the male fertility of the original bisexual form in the polymorphic population be changed from $b$ to $(1-C) b$, by a mutation having the same proportionate effect on selfing. As before, the fitness of the new form is given by Eq. (1), which in the present notation gives:

$$
\left\{(1+c)[1+s(1-C)(1-2 \delta)]+\frac{U b}{V}(1-C)\right\} / 2 W
$$

where $c$ is the increase in female fertility of the new form relative to that of the original type, and $U, V$ and $W$ are those for the polymorphic population, before introduction of the second male-fertility mutation. The condition for the new form to have an advantage in comparison with the more male fertile form is, as before, that the above fitness expression must exceed 1. Using the property that at equilibrium before the new form arises, the fitness of the more male-fertile form is 1 , we obtain the condition;

$$
c>\frac{C[(U b / V)+s(1-2 \delta)]}{1+(1-C) s(1-2 \delta)} .
$$

(This condition is valid if $s \delta<\frac{1}{2}\left(\frac{U b}{V}+s\right)$, which takes its smallest value, $\frac{1}{2}$, when "females" are absent; when $s \delta$ exceeds this value, reducing the 
selfing rate is highly advantageous, and the new form can spread even if it does not increase female fertility.) From (la), the values of $U, V$ and $W$ are as follows:

$$
\begin{aligned}
U & =(1-s)(1-p)+\left(1-s_{f}\right)(1+k) p \\
V & =b(1-p)+(1-K) b p \\
W & =(1-s \delta)(1-p)+\left(1-s_{f} \delta\right)(1+k) p
\end{aligned}
$$

where $p$ is the frequency of the "female" form in the polymorphic population. It is easily shown that if $s_{f}<s, U b / V$ is an increasing function of $p$; the condition given above is therefore harder to satisfy for a population at equilibrium with "females" present than in the original population. Table 3 shows some numerical examples of the condition for the spread of a second mutation halving male fertility $(C=0.5)$, assuming that one such mutation has occurred previously and reached equilibrium in the population. The minimum increases in female fertility are never less than those for the corresponding cases in table 2.

So far we have only studied the conditions for male-fertility mutations to be advantageous in the original, male-fertile form. We must also consider the possibility that further reductions in male fertility, even though disadvantageous in the bisexual form, might be favoured in the "females" sufficiently to ensure the spread of such genes in populations polymorphic for "female "forms. It is easy to show by the method used above that the condition for a reduction in the male fertility of the "female" to be selectively advantageous is easier to satisfy than the condition given above for the bisexual form; this is illustrated in table 3. However, in contrast to the result for the bisexual form, the minimum necessary increase in female fertility may sometimes be less when a gene reducing male fertility is introduced into the "female" form than when introduced into the original population, before the female form arose. When a "female" type has increased to fixation, it can be shown that a second mutation with the same phenotypic effects is always less easy to establish than the first. It is therefore not impossible, though unlikely, that a gradual progress towards gynodioecy could take place. In cases when a mutation reducing male fertility is advantageous in the "females", but disadvantageous in the more male-like form, establishment will often be more likely if the mutant locus is linked to the first male-fertility mutation.

\section{(ii) Reduction in female fertility}

Now suppose that a mutation occurs reducing female fertility to $1-c$ of the previous value; let the effect on male fertility be a relative increase of $C$. The fitness of this new phenotype is, by Eq. (1):

$$
\left\{(1-c)\left[1+s_{m}(1-2 \delta)\right]+\frac{U b}{V}(1+C)\right\} / 2 W
$$

where $s_{m}$ is the selfing rate of the new form. In a monoecious species, this type of change could be produced by a mutation increasing the ratio of male 
to female flowers. It seems unlikely that the selfing rate of a form with reduced ovule production would be lower than that of the original form. If we assume that $s_{m}=s$, we obtain the following condition for the spread of a mutation reducing female fertility:

$$
C>\frac{V}{U b} \times c[1+s(1-2 \delta)]
$$

If the starting population is homogeneous for the original, fully male fertile form the values of $U, V$ and $W$ are as in Eqs. (2), and we have:

$$
C>\frac{c}{1-s}[1+s(1-2 \delta)]
$$

which gives the condition for the spread of a mutant with lowered female fertility. The analogous condition for a mutant with lowered male fertility is condition (6). When $s=0$, the two conditions are the same, once put in the same notation $(k=c, K=C)$ : for a reduction in male fertility to spread, the female fertility must be increased by more than the reduction in male fertility, while a mutation reducing female fertility will spread only if it causes a more than proportionate increase in male fertility. As in the case of complete sterility mutations (Lloyd, 1975c; Charlesworth and Charlesworth, 1978), when there is some selfing the condition for spread of a mutation reducing male fertility becomes easier, while that for a reduction in female fertility becomes harder to satisfy. In quantitative terms, this effect is smaller for partial effects on male or female fertility. Also, condition (9) shows that if $\delta=1$ the chance of spread of a female-fertility mutant is the same regardless of selfing rate. It is thus possible that under some conditions the spread of genes for partial reduction in female fertility could be only slightly less probable than for reduced male-fertility mutations, but there will always be some differential in favour of male sterility mutations.

Condition (8) can also be used to study the possibility of the spread of mutations reducing female fertility in populations that are polymorphic for male-fertility genes. As discussed in the previous section, the term $V / U b$ decreases with frequency of "females". This means that the conjecture of the introduction to Section 4 is confirmed: the male-fertile form is more likely to be changed in the direction of maleness if females are present in the population, than if they are absent. Similar conclusions are reached in the case of complete female sterility (i.e. $c=1, s_{m}=0$ ). In this case too, there must be some increase in male fertility for the new form to have an advantage. The critical value of $C$ depends on $c, s, \delta$ and on the parameter values assumed for the female-like form; once again it decreases with increasing frequency of "females".

The general case, when reduction in female fertility is accompanied by an effect on the selfing rate, is more complicated, and the results depend on the inbreeding depression. If $\delta>\frac{1}{2}$, there is a condition on $C$ only when $s_{m}$ exceeds :

$$
\frac{c+s(1-2 \delta)}{(1-c)(1-2 \delta)}
$$

which is less than $s$. If there is such a condition, the minimum value of $C$ is again found to decrease, the commoner the female form in the starting 
population. If $\delta<\frac{1}{2}$, the form with reduced female fertility can have an advantage even if $s_{m}$ is less than $s$, provided that $C$ exceeds a minimum value which decreases as "females" become commoner; if $s_{m}$ is greater than the value given above, however, there is no restriction on $C$.

A similar treatment of the fitness of the "female" form, if modified by the same mutation reducing female fertility, shows that this would be lower than the value given by Eq. (7), and that the analogous condition to (8) for the mutation to have an advantage in the "females" is

$$
C>\frac{V}{U b} \times \frac{c(1+k)[1+(1-K) s(1-2 \delta)]}{1-K}
$$

which can be shown to be more stringent than (8). In other words, a female-sterility factor may have an advantage in one form of the starting population, but a disadvantage in the other. Table 3 shows some numerical illustrations of the conditions for reduced female fertility to be favoured in the two phenotypes in a population polymorphic for male-fertility genes. For the same rate of selfing of the less female-fertile form, the minimum $C$ values are lower, the higher the increase in female fertility in the "female" form. When $\delta$ exceeds $\frac{1}{2}$, we find, as expected, that if the female-sterility mutation causes an increase in the selfing rate, the minimum values of $C$ are higher than if it does not. The lower part of the table shows the opposite effect when $\delta<\frac{1}{2}$. In all cases, the minimum increases in male fertility that will give the mutation an advantage are higher in the "female" than in the bisexual form.

We have done computer calculations of the progress of mutations that reduce female fertility, introduced into populations polymorphic for "females". We have shown above that the fitness of the "females" may be lowered by a change that gives the original bisexual form an increased fitness. It is therefore likely that the second gene will be able to increase in frequency only if it is linked to the male-fertility locus. This is frequently found in the results of our computer runs.

\section{Disaussion}

\section{(i) Effects of reducing male fertility}

The fate of a gene conferring complete male sterility depends on the interaction of two factors, the amount of inbreeding depression, which determines the advantage of avoiding self-fertilisation, and the extent of reproductive compensation (increase in female fertility when male fertility is abolished, or vice versa). When considering the fate of a phenotype with a partial reduction in male fertility, matters become complicated by yet a third factor, the "genetic value" of pollen. It has long been understood that pollen has a lower genetic value in a selfing species than in an outcrossing one, and that natural selection will tend to promote a decrease in the quantity of pollen that is produced by a species that is predominantly selfing. Such differences have been noted by botanists in a number of selfing species, for instance by Baker (1965) in a comparison of (selfing) 
weeds with closely-related non-weedy species. Cruden (1977) reviews a number of such cases. When we examine the conditions for the spread of partial male-sterility genes, as in the present paper, we must therefore bear in mind that such genes may be favoured even if they do not affect the rate of selfing. It is immediately seen from condition (4) of Section 3 above that if the inbreeding depression is less than $\frac{1}{2}$, the increase in female fertility that is necessary to ensure the advantage of a reduction in male fertility is less if it does not affect selfing, than if it decreases the selfing rate; the opposite is true if the inbreeding depression exceeds $\frac{1}{2}$.

\section{(ii) Selection for reduced male fertility in monoecious and hermaphrodite species}

The model studied in this paper was developed primarily to explore the possibility of the evolution of dioecy from monoecy. As discussed in Section 4 (ii) above, mutations producing male-like forms are less likely to spread than ones producing partial or total females. The first question to be discussed is therefore whether the conditions for the spread of genes reducing male fertility can be met in monoecious species.

In monoecious species, selfing rates would presumably be lower than in self-fertile hermaphrodites. Lloyd (personal communication) has found this to be true for the respective monoecious ancestors of Cotula pyrethrifolia and $C$. dendyi. It is thus unlikely that the product of the selfing rate and the inbreeding depression could exceed $\frac{1}{2}$. There is some evidence for, though no good quantitative estimates of, inbreeding depression in monoecious species. It is well known that it occurs in maize, which was one of the species originally studied by Darwin (1876). Other than this example, the only estimate of inbreeding depression in a monoecious plant appears to be that of Lloyd (1972). Using data from three Cotula species, the average seed set after self-pollination was 72 per cent of that on cross-pollination; there were also large differences in germination percentages, but not in survival after germination. These data support the assumption that a monoecious species might show marked inbreeding depression. We have shown above that, with $s \delta<\frac{1}{2}$, some increase in female reproduction must be assumed if a "female" form is to be established in a monoecious species.

In a monoecious plant, reduction of male fertility could occur in either of two ways: the mutants could produce a smaller quantity of effective pollen, or some male flowers might be converted to female flowers. The first possibility seems less likely for a monoecious species than a hermaphrodite, for the following reasons. If a mutation simply causes loss of pollen function, or decreases the amount of pollen, the amount of extra resources made available in a monoecious plant would be small, since the male flowers would still be produced, and the energy involved in making the pollen is presumably only a fraction of that required for the complete flower. In a hermaphrodite flower it might be possible for the energy saved by reducing pollen output to be diverted to female reproduction within the same flower. In a hermaphrodite, partial male sterility due to this type of mutation would probably not be advantageous, since the selfing rate would be unlikely to be much affected unless pollen production were greatly reduced. As shown in Section 3, for genes reducing male fertility to be advantageous, selfing must be reduced by more than some critical amount (not necessarily as great as the reduction in male fertility-see table 2). 
Thus we would expect that hermaphrodite species would become polymorphic only for genes causing substantially reduced male fertility, and this is of course the situation in gynodioecious species, which consist of hermaphrodite and female individuals.

Reduced male fertility with a substantial effect on selfing could be achieved in a hermaphrodite by means of a mutation converting some flowers into females. Fixation of such a gene would produce gynomonoecy, which is known in many species, for example among the Compositae.

The second type of mutation that could occur in monoecious species, i.e. conversion of male into female flowers, has been observed in maize. It has occurred at several loci, as both dominant and recessive mutations, called "tassel-seed" (Jones, 1934). Such a change automatically ensures an increase in female fertility, which could be considerable if male flowers are commoner than female. There thus seems no reason why a completely female form should not spread in a monoecious species, giving the monoecious analogue of gynodioecy. Such cases, however, seem to be much rarer than true gynodioecy. G. Valdeyron and D. G. Lloyd (personal communication) have studied a case in Ficus carica. Partial changes of this sort could be advantageous, since selfing is likely to be considerably reduced, by up to as much as the reduction in male fertility itself, so that such mutations could spread if inbreeding depression exceeds one-half.

Whatever the nature of the phenotypic effects of a mutation reducing male fertility, we have shown that small changes are likely to become fixed, while mutations with larger effects may establish polymorphism. Thus hermaphrodite species might become gynodioecious, gynomonoecious, or polymorphic for gynomonoecy (though no case of this last is known to us), and monoecious species might become polymorphic for females or for partial females, or might simply become fixed for a new ratio of male to female flowers. When a male-sterility gene, or one with a partial effect, has become fixed in a population, the spread of another such mutation is harder than before (see Section 4). The same is usually true when a form with reduced male fertility has become polymorphic, though further reduction of the male fertility of the "female" form in such a population may sometimes be favoured more than the first such change. Incorporation of a long succession of mutations, leading finally to gynodioecy, is therefore unlikely. Two cases are known, however, where more than one gene reducing male fertility may have been incorporated. In the gynodioecious species Silene maritima (Baker, 1966) and in Origanum vulgare (Kheyr-Pour, 1975), a variable fraction, around 5 per cent, of the pollen-bearing individuals carry a mixture of female and hermaphrodite flowers. It would be interesting to know the genetics of these cases, as it is possible that they are merely due to incomplete penetrance of the genes causing complete male sterility in the females.

If a mutation causing a slight loss of male fertility becomes fixed in a population, a subsequent mutation of the same sort is more likely to establish a polymorphism. This is easy to demonstrate for the case when there is no compensation: once a gene reducing the selfing rate has been fixed, the condition for polymorphism given in Section 3 is easier to satisfy, since the value of $s$ is lower. Similarly, assuming that the mutations decreasing male fertility also increase female fertility, table 2 shows that a given reduction in selfing rate may go to fixation when $s$ is high, but become polymorphic when $s$ is lower. 


\section{(iii) Selection for increased male fertility in populations containing female forms}

Assuming the presence of female-like individuals in a population, we have shown that mutations making the bisexual form more male-like can enter the population more easily than before (Section 4 (ii)). When no "females" are present, a mutation abolishing female fertility can spread only if it causes a considerable increase in male fertility (Lloyd, 1975c; Charlesworth and Charlesworth, 1978), so that androdioecy is extremely unlikely to evolve. Partial reduction in female fertility is subject to similar constraints, and would also be hard to establish in populations. Nevertheless, andromonoecy is known in plants, for example in several species of Umbelliferae, and may well be at least as common (Lloyd, personal communication). Since andromonoecy is unlikely to evolve as an outbreeding device, as discussed above, other factors must be more important, such as factors ensuring especially low genetic value of the ovules that have become male. For example, the male flowers in some Umbellifers are large, showy florets at the edge of the inflorescence; if these flowers had already been selected for decreased ovule output as a necessary concomitant of increased petal size, such an especially favourable situation could exist. Whatever the details of particular cases, additional factors as well as those in the models presented in this paper are likely to be involved. This would make studies of andromonoecy particularly valuable, since they could give information about such questions as the energy costs of male and female reproduction.

Returning to the case of a population polymorphic for "females", we have shown in this paper that reduction in female fertility is more advantageous in the bisexual form than in the "females". If compensation is sufficient for the mutation to be advantageous in bisexual individuals, it may nevertheless be disadvantageous in the "females", and thus may be unable to spread unless it is linked to the male-fertility locus. The existence of such a "linkage constraint" has been confirmed by computer runs. Dioecy evolved by the incorporation of an alternating sequence of mutations affecting male and female fertility would thus be controlled by a set of linked genes. Recombinant types would be present unless linkage was extremely tight, so that both males and females with variable traces of opposite-sex function would be found. This is exactly the situation found in Cotula by Lloyd (1975a), and contrasts with the situation when dioecy has evolved via gynodioecy, where only " males" will vary in their sex phenotype. This has been observed in several species that are classed as dioecious or gynodioecious, e.g. Cirsium arvense (Correns, 1916; Lloyd and Myall, 1976), Carex picta (Martens, 1939), see also Westergaard (1958).

The achievement of dioecy by means of a series of mutations, gradually reducing male or female fertility would probably be an extremely slow process. Not only would time be required for mutations to arise with sufficient compensation effects, and be incorporated in the population, but the correct dominance and linkage relations would have to be met. Intermediate states should therefore be common. This pathway has been termed "paradioecy" (Lloyd, 1973). On the model developed in the present paper, one would expect "females" to be more unisexual than "males", though each would show some degree of opposite-sex function. Lloyd (1978 and personal communication) has found this to be the case in Cotula pyrethrifolia and $C$. dendyi. 


\section{(iv) Evolution of monoecy}

A further question that may be considered in relation to the topics discussed here is the evolution of monoecy. The flowers of monoecious species frequently show traces of opposite-sex function (Darwin, 1876), which suggests that monoecy has evolved from hermaphroditism. This agrees with the distribution of monoecy. For example, among the British Composites there are seven monoecious species out of 173 , and these are found in five genera. Like dioecy, monoecy must be separated by at least two genetic changes from hermaphroditism. Gynomonoecy or andromonoecy would occur as an intermediate stage. In the British Composites, there are 99 gynomonoecious species, but all are in genera that have no monoecious members. There are, however, at least three mixed genera among the New Zealand Composites (Lloyd, personal communication). If we assume that this is the usual route, the problem of the evolution of monoecy reduces to the question of how the hermaphrodite flowers of a gynomonoecious species could become converted into male flowers. This is a case of reduction of female fertility, which is studied in Section 3 (ii), above.

Using Eq. (7), the general formula for the fitness of a form with reduced female fertility, and assuming that it is introduced into a gynomonoecious population with selfing rate $s$, we obtain the condition for spread that the increase in male fertility $C$ must exceed $A /(1-s)$ where $A$ is given by:

$$
c\left[1-s_{m}(2 \delta-1)\right]+\left(s-s_{m}\right)(2 \delta-1)
$$

and $c$ is the reduction in female fertility. If both types of flowers produce the same number of ovules per flower, $c$ is simply the frequency of hermaphrodite flowers in the gynomonoecious state. We shall discuss this assumption further below. Now the results of Section 3 and Table 2 show that fixation of a mutation that causes some flowers of a hermaphrodite to become female, thus producing gynomonoecy, would be unlikely to occur if the frequency of hermaphrodite flowers were reduced much below $0 \cdot 5$. We must therefore assume that $c$ would be at least 0.5 after this initial step.

It is likely that the new, monoecious, form would have a lower selfing rate than the original type, with hermaphrodite and female flowers. If we write $s_{h}$ for the selfing rate of a hermaphrodite flower in a gynomonoecious species, and assume that the female flowers have a selfing rate of $s_{m}$, the same as in monoecy, we can obtain a value for the selfing rate of the gynomonoecious species, as follows:

$$
s=s_{m}(1-c)+s_{h} c, \quad s_{h}>s>s_{m} .
$$

Condition (10) then reduces to:

$$
\frac{C}{c}>\frac{1-s_{h}(2 \delta-1)}{1-s}
$$

It is also easy to show, by the same method as in Section 3, assuming full dominance or recessivity, that the condition for fixation, rather than polymorphism, is:

$$
\frac{C}{c}>\frac{1+C}{1-c} \times \frac{1-s_{h}(2 \delta-1)}{1-s_{m}} .
$$


These conditions show that if $s_{h}$ and $\delta$ are close to 1 , the fixation of genes converting hermaphrodite flowers into males is, as one might expect, possible with very little increase in male fertility. Although it may be reasonable to assume that $s_{h}$ is nearly $1, \delta$ would probably be smaller than this.

Table 4 shows some numerical examples of the ranges of $C$ that are compatible with polymorphism. Clearly, fixation becomes more likely, the higher the value of $\delta$, the greater the effect in terms of reduction of selfing (i.e. the lower $s_{m}$ ), and the lower $c$. The conditions that the inbreeding depression should be high and $s_{m}$ low are not implausible. The condition that $c$ must be low, or else polymorphism will occur, is more problematical.

\section{TABLE 4}

Range of values of $\mathrm{C}$, the increase in male fertility, that will give a polymorphism for a phenotype with female fertility $1-\mathrm{c}$, and selfing rate $\mathrm{s}_{\mathrm{m}}$. When an upper limit is not shown, fixation cannot occur. The value of $\mathrm{s}_{\mathrm{m}}$ is assumed equal to $0 \cdot 8$

The values of $s$, from Eq. (11) are

\begin{tabular}{|c|c|c|c|c|}
\hline & & & & \\
\hline & $s_{m}$ & $0 \cdot 2$ & 0.5 & \\
\hline & 0.1 & 0.24 & 0.45 & \\
\hline & 0.5 & 0.56 & 0.65 & \\
\hline Inbreeding & & & & \\
\hline & $s_{m}$ & & & 0.5 \\
\hline 0.9 & $0 \cdot 1$ & 0.09 & 111 & $0.327-2 \cdot 000$ \\
\hline 0.9 & 0.5 & 0.16 & 20 & $0.514-2.571$ \\
\hline 0.6 & 0.1 & 0.22 & 304 & $0.764-14.00$ \\
\hline 0.6 & 0.5 & 0.38 & 24 & $1 \cdot 200$ \\
\hline 0.4 & $0 \cdot 1$ & 0.30 & 775 & $1 \cdot 055$ \\
\hline $0 \cdot 4$ & 0.5 & 0.52 & 381 & $1 \cdot 657$ \\
\hline $0 \cdot 1$ & $0 \cdot 1$ & 0.432 & 37 & $1 \cdot 491-$ \\
\hline 0.1 & 0.5 & 0.746 & 556 & $2 \cdot 343$ \\
\hline
\end{tabular}

If it is correct to equate $c$ with the frequency of hermaphrodite flowers in the original gynomonoecious population, we have already given reasons why $c$ would rarely be expected to be below $\frac{1}{2}$. However, $c$ will be lower than the frequency of hermaphrodite flowers if these have lower ovule output than the female ones, in the original gynomonoecious state. For example, if the frequency of hermaphrodite flowers is $0 \cdot 6$, but the ovule output is 50 per cent higher than that of the hermaphrodite flowers, $c$, the loss of female fertility when all hermaphrodite flowers are converted to males, is only 0.5 ; if the ovule output of female flowers is trebled, $c$ is only 0.333 , but it seems improbable that the loss of pollen in the female flowers could give such a large increase in their female fertility.

One possibility that must be considered here is that, before the final mutation producing full monoecy, the gynomonoecious population may first be selected for partially decreased female fertility of the hermaphrodite flowers. Such changes would of course involve only small $c$ values, and could readily be fixed, provided that male fertility was increased, as seems likely. Even if such a change causes an increase in the selfing rate of the hermaphrodite flowers, it need not necessarily increase the overall selfing 
rate, if the female fertility of the hermaphrodite flowers is decreased enough. Once a mutation producing such changes has been incorporated into a gynomonoecious species, the conditions specified above for the fixation of a gene making the hermaphrodite flowers male, thus giving complete monoecy, are more easily satisfied than before.

This line of argument leads to the conclusion that the evolution of monoecy would be expected to involve at least two genetic changes, but more likely three or more, first a mutation converting some flowers into females, and then two or more mutations reducing the female fertility of the remaining hermaphrodite flowers. Except while the genes concerned were spreading to fixation, the populations would be genetically homogeneous. There would therefore be no tendency for linkage between the genes involved. There appear to be no data about the inheritance of monoecy. It would be interesting to have such information, which could serve to test the multi-gene model of the evolution of monoecy. This might be a more favourable case for study than dioecy, where tight linkage is expected (see above), and where the involvement of more than one gene is therefore harder to demonstrate. On the other hand, it may be that no species with both monoecious and hermaphrodite races exist; this is very likely to be the case if the evolution of monoecy requires the fixation of three or more mutations.

It seems, from the above considerations, that monoecy will evolve in certain restricted circumstances. It would therefore be expected to be rare. Again, this prediction is hard to test, but seems to be supported in the British flora where there are only four families containing both hermaphrodite and monoecious species (Chenopodiaceae, Alismataceae, Araceae and possibly Rosaceae) and two with hermaphrodite, monoecious and dioecious species, compared with nine families with hermaphrodite and dioecious species. At the generic level, only Poterium is a possible case of monoecy together with hermaphroditism, whereas there are six sporadic dioecious members of hermaphrodite genera.

Finally, it should be pointed out that our model involves the assumption that monoecy evolves in self-compatible species, essentially as a means of protection against inbreeding depression. Any cases of self-incompatible monoecious species clearly cannot be accounted for on this basis. A number of such cases are known to exist (Godley, 1955).

Acknowledgments.-We are very grateful to D. G. Lloyd and J. J. Bull for reading and commenting on the first draft of this manuscript, and to D. G. Lloyd and G. Valdeyron for suppying data from their unpublished studies of Cotula and Ficus.

\section{REFERENCES}

BAKER, H. G. 1959. Reproductive methods as factors in speciation in flowering plants. Cold Spr. Harb. Symp. Quant. Biol., 24, 177-191.

BAKER, H. G. 1965. Characteristics and mode of origin of weeds. In The Genetics of Colonising Species, eds. H. G. Baker and G. L. Stebbins. Academic Press, New York. BAKER, H. G. 1966. The evolution of floral heteromorphism and gynodioecism in Silene maritima. Heredity, 21, 689-692.

CHARLESWORTH, B., AND CHARLESWORTH, D. 1978. A model for the evolution of dioecy and gynodioecy. Amer. Nat., in press. 
CORRENS, c. 1916. Untersuchungen über Geschlechtsbestimmung bei Distelarten. Sber. Preuss. Akad. Wiss., 20, 448-477.

GRUDEN, R. w. 1977. Pollen ovule ratios: a conservative indicator of breeding systems in flowering plants. Evolution, 31, 32-46.

DARWIn, c. 1876. The Effects of Cross and Self Fertilisation in the Vegetable Kingdom. John Murray, London.

Darwin, c. 1877. The Different Forms of Flowers on Plants of the Same Species. John Murray, London.

GoDley, E. J. 1955. Monoecy and incompatibility. Nature, 176, 1176-1177.

JONES, D. F. 1934. Unisexual maize plants and their bearing on sex differentiation in other plants and in animals. Genetics, 19, 552-567.

KHeyr-Pour, A. 1975. Déterminisme génétique et répartition écologique de la stérilité mâle cytoplasmique chez Origanum vulgare L. Thesis, Univ. des Sciences et Techniques de Languedoc, Montpellier.

LEWIS, D. 1942. The evolution of sex in flowering plants. Biol. Rev., 17, 46-67.

LLOYD, D. G. 1972 . Breeding systems in Cotula. II. Monoecious populations. New Phytologist, 71, 1195-1202.

Lloyd, D. G, 1973. Sexual dimorphism in Cotula (Compositae). (Abstr.) Genetics, 74, s161. LLOYD, D. G. 1974. Theoretical sex ratios of dioecious and gynodioecious angiosperms. Heredity, 32, 11-34.

Lloyd, D. G. 1975a. Breeding systems in Cotula. III. Dioecious populations. New Phytologist, 74, 109-123.

LLOYD, D. G. 1975b. Breeding systems in Cotula. IV. Reversion from dioecy to monoecy. New Phytologist, 74, 125-145.

LLoYD, D. G. 1975c. The maintenance of gynodioecy and androdioecy in angiosperms. Genetica, 45, 325-339.

LLOYD, D. G. 1978. The distribution of gender in four representative angiosperms. Unpublished manuscript.

Lloyd, D. G., AND MYAlL, A. J. 1976. Sexual dimorphism in Cirsium arvense (L.) Scop. Ann. Bot., 40, 115-123.

martens, J. L. 1939. Some observations on sexual dimorphism in Carex picta. Amer. J. Bot., 26, 78-88.

WESTERGAARD, м. 1958. The mechanism of sex determination in dioecious flowering plants. Adv. Genet., 9, 217-281. 